\title{
A Reduced-Order Modeling Framework for Integrated Thermo-Elastic Analysis of Hypersonic Vehicles
}

\author{
Nathan J. Falkiewicz ${ }^{1}$ and Carlos E.S. Cesnik ${ }^{2}$ \\ Department of Aerospace Engineering, University of Michigan, Ann Arbor, MI, 48109-2140, USA
}

\begin{abstract}
This paper presents an integrated, reduced-order thermo-elastic modeling framework for fundamental characterization of the impact of aerodynamic heating on the structural dynamics and controllability of hypersonic vehicle's structures. The emphasis in this methodology is to obtain structural modes and frequencies as a function of the thermal boundary conditions and initial conditions without having to solve the full-order thermoelastic problem at every time step. This is motivated by the fact that control-oriented analysis and vehicle design require solution techniques that are computationally efficient and possess a low number of states. Knowledge of the transient structural dynamics over a flight trajectory will support the fully coupled aerothermoelastic/propulsion analysis and will enable the investigation of the movement of the poles and zeros of the linearized flight dynamics for determination of the required robustness of the flight control system. Proper Orthogonal Decomposition (POD) is proposed for solution of the transient heat transfer problem. A structural dynamics solution procedure is outlined in which thermal effects are coupled to the structural dynamics through the discrete form of the Ritz method. Due to the high temperatures expected in hypersonic flight, the equations of motion include a temperature-dependent Young's Modulus and the geometric stiffness matrix due to thermal stresses which will lead to coupling of the equations of motion. The methodology is exemplified using a representative hypersonic vehicle control surface as this structure is believed to have potentially the greatest impact on the dynamics of the vehicle.
\end{abstract}

\section{Introduction}

$\mathrm{T}$ HERE has recently been renewed interest in hypersonic vehicles (HSVs), particularly for rapid response to global threats and affordable access to space. However, there still exist inherent challenges associated with hypersonic flight due to strong interactions among aerodynamics, heat transfer, elastic airframe, propulsion system, flight dynamics, and controls. This work focuses on the coupling of heat transfer and structural dynamics of the vehicle. Due to the high speed of HSVs, aerodynamic heating will occur at the vehicle surface, leading to heat transfer through the internal vehicle structure. The resulting change in the temperature distribution throughout the structure will lead to a change in its structural dynamics through various phenomena to be described in subsequent sections. It is important to be able to predict the transient thermal response and resulting structural dynamic characteristics over the flight trajectory due to the coupling described above. Specifically, deflection of the forebody alters the angle-of-attack and thus the airflow through the engine, resulting in changing thrust. ${ }^{1}$ Additionally, the transient structural dynamics can lead to evolution in vehicle control characteristics which determine the necessary robustness of the control system. ${ }^{1}$ In the conceptual design phase, these complex interactions among disciplines lead to the need for modeling techniques which can predict the thermal response and resulting structural dynamics within a vehicle design and control analysis framework.

One modeling technique that is traditionally used for thermo-elastic problems involves the use of high-fidelity finite element models. Solution of the coupled thermo-elastic problem in a high-fidelity sense for this application begins by determining the heat flux at the vehicle surface due to aerodynamic heating at some initial cruise configuration. Based on the heat flux boundary condition at the surface, the temperature distribution throughout the structure can then be calculated from transient thermal finite element analysis. Once the temperature distribution is

\footnotetext{
${ }^{1}$ Graduate Student Research Assistant, Dept. of Aerospace Engineering, University of Michigan, 1320 Beal Ave, Ann Arbor, MI 48109, Student Member AIAA.

${ }^{2}$ Professor of Aerospace Engineering, Dept. of Aerospace Engineering, University of Michigan, 1320 Beal Ave, Ann Arbor, MI 48109, Associate Fellow AIAA.
} 
found at the time instant of interest, two modifications to the structural stiffness need to be considered - one due to the temperature dependence of Young's Modulus and the other due to the effect of thermal stresses. The former can be calculated by directly modifying the structural stiffness matrix based on a functional dependence of Young's Modulus with temperature. The latter contribution can be found by solving a static problem subjected to thermal loads, calculating the thermal stresses, forming the geometric stiffness matrix, and adding the geometric stiffness matrix to the structural stiffness matrix. Once these modifications are made to the stiffness at the time instant under consideration, solution of the structural dynamics problem yields vibration modes and frequencies of major structural components at that time instant. The heat flux at the next time step can then be found from aeroheating analysis based on deflections at the current time step and the loop can be repeated. The deflections at the current time step can also be used in an aerodynamic representation to recalculate the surface pressures on the vehicle and update the load vector in the structural finite element model. A schematic of the high-fidelity solution process as described above is shown in Fig. 1.

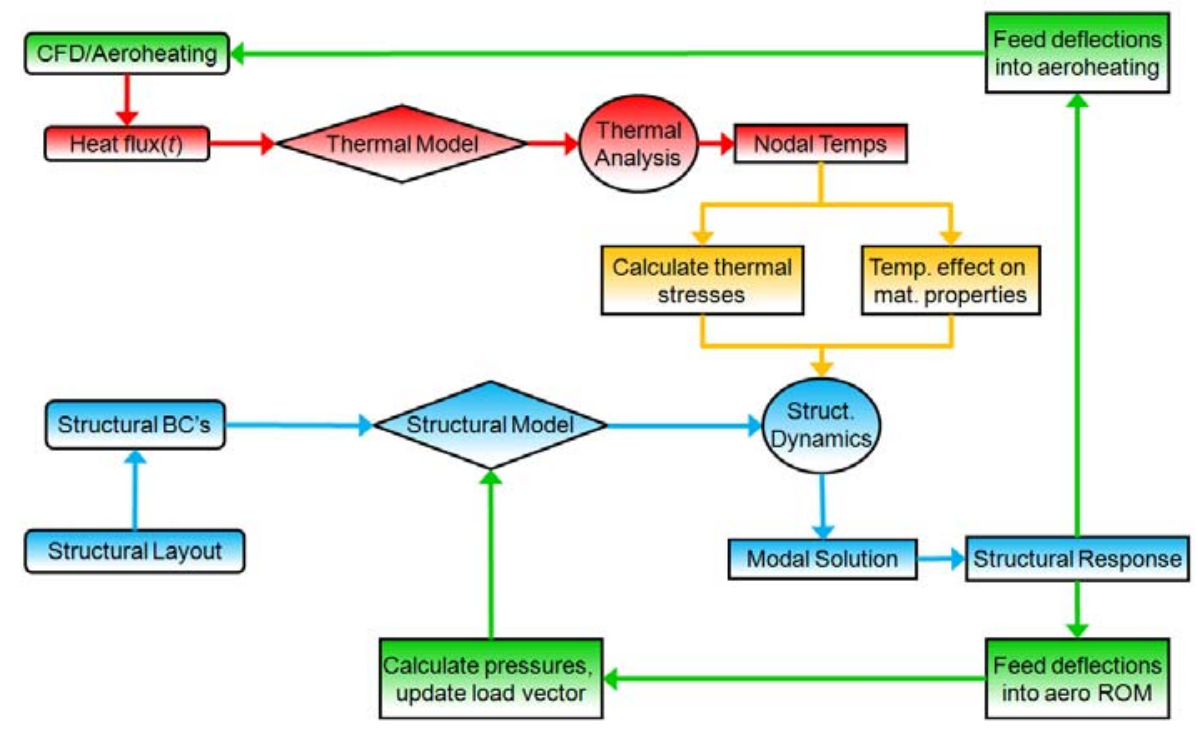

Figure 1. High-fidelity coupled thermo-elastic solution process.

A similar high-fidelity finite element procedure was employed by Lindell and Amundsen ${ }^{2}$ to analyze the static stresses due to thermal loads on the Hyper-X vehicle. This approach made use the SHABP aeroheating code and Fay-Riddell stagnation point heating theory to determine the aerodynamic heat flux at the vehicle surface based on trajectory information. Patran Thermal was used to carry out transient thermal analysis to determine the temperature distribution based on the given boundary conditions over the flight trajectory. Iteration was carried out between the aeroheating analysis and heat transfer analysis to capture the correct skin temperature of the structure. Temperatures were interpolated from the thermal grid to the structural grid and nonlinear static structural finite element analysis was performed at discrete trajectory points. The thermal analysis included radiation to the atmosphere, radiation within cavities, and contact resistance between parts and across welds. The structural analysis included temperaturedependent Young's Moduli, coefficients of thermal expansion, and stress/strain curves. Orthotropic material properties were also included where applicable. Results of the thermal analysis of a control surface showed maximum temperatures over 3,000 degrees Fahrenheit at the end of the predicted transient. Nonlinear static structural analysis based on the predicted thermal loads showed a maximum strain of $-0.69 \%$ which occurred in the skin region. An initial yield assessment based on a temperature-dependent yield stress showed regions in which the Von Mises stress was up to three times the local yield stress value.

While the above high-fidelity finite element technique provides an accurate representation of the effects of aeroheating and heat transfer on the static structure, this method would be too complex and possess too many states for the purpose of control simulation and vehicle design. A model reduction technique proposed by Collier ${ }^{3}$ involved the use of equivalent stiffness and thermal coefficients for analysis of stiffened plates via classical lamination theory. The goal of that work was to reduce a three-dimensional stiffened panel to a homogenized two-dimensional planar finite element model having equivalent structural stiffness and thermal expansion for static structural analysis. This formulation sought to reduce the number of degrees of freedom by avoiding the need to generate a high-fidelity mesh of the actual three-dimensional structure, and replace it with a two-dimensional planar mesh. Based on the 
given stiffener geometry and spacing, an equivalent membrane stiffness matrix, $A$, membrane-bending coupling matrix, $B$, and bending stiffness matrix, $D$, were calculated. To capture the thermal expansion of the actual panel, an equivalent membrane thermal coefficient matrix, $A^{\alpha}$, membrane-bending coupling thermal coefficient matrix, $B^{\alpha}$, and bending thermal coefficient matrix, $D^{\alpha}$, were also calculated. These resulting matrices were input into the material property definition in the finite element software to define the equivalent anisotropic plate element for thermoelastic analysis. Comparison of the stiffness terms from the homogenized formulation with those from threedimensional finite element analysis of the actual plate showed good agreement. Additionally, comparison of the thermal stress resulting from an assumed temperature distribution between the homogenized formulation and threedimensional finite element analysis also showed good agreement.

While the above methodology was able to reduce the number of degrees of freedom in the static structural solution of reinforced panels, it still did not address the dynamics of complex structural constructions. An attempt to include dynamic effects comes from Culler, Williams, and Bolender. ${ }^{4}$ The authors made use of the assumed mode method to analyze the effect of propellant consumption and heating on the mode shapes and natural frequencies of a free-free beam representative of a HSV. For the thermal problem they considered one-dimensional heat transfer through the thickness of the beam. The structure was taken to be composed of an outer PM 2000 honeycomb sandwich panel, an insulating layer made of IMI Insulation, and a load-carrying member made of Titanium. The heat flux boundary condition due to aerodynamic heating at the surface was calculated using the Eckert reference temperature method for turbulent, high speed flow over a flat plate based on hypersonic cruise of 2,000 psf dynamic pressure at 85,000 feet. The structural dynamics were calculated using an analytical formulation of the free-free beam equations in which assumed free-vibration modes were employed to generate natural frequencies and vibration mode shapes over the flight trajectory. The authors investigated both the effect of mass change due to propellant burn and material degradation due to aerodynamic heating on the structural dynamics. Results showed that the combined effect of mass decrease and temperature increase resulted in a small change in the first bending mode frequency, but a significant change in the second and third bending mode frequencies. Because a uniform temperature change with no spatial variation did not affect the mode shapes, evolution of the mode shape over time was governed by the mass decrease. Analysis of the resulting flight dynamics showed that aerodynamic heating did not significantly affect the rigid body poles and zeros due to the fact that the rigid body and flexible states are not excited by each other. The short-period poles were found to remain somewhat constant during hypersonic cruise, but movement of the real transmission zeros to the right resulted in more available bandwidth to the inner-loop control law. Finally, it was noted that the first bending frequency was significant in terms of aeroelastic interactions because it lies near the elevator actuator bandwidth. Further analysis of the aeroservoelastic interactions at various flight conditions was proposed. That study, however, was limited to the HSV body only, and no other surfaces were considered (e.g., control surfaces). Moreover, the beam representation of the airframe, although able to capture its basic structural dynamic characteristics, was too coarse to model the thermal and mechanical spatial distribution and corresponding effects on the structural response.

As opposed to the above fundamental analytical model for the structural dynamics, the method described in this work begins with a high-fidelity representation of the structure and then systematically reduces the order of the system. Additionally, this work will consider heat conduction in three dimensions. A thermal solution scheme based on Proper Orthogonal Decomposition (POD) is proposed for solution of the transient heat transfer problem. The discrete form of the Ritz method with modal truncation will be used for solution of the structural dynamics problem. The fact that ROMs will be extracted from high fidelity three-dimensional models provides various advantages. Because the ROMs are generated based on output of detailed numerical models, they are independent of geometry and can be easily updated for parametric studies. In contrast to fundamental models, modeling as much physics as possible initially and then reducing the order will allow one to control the error incurred due to model reduction through variation of certain parameters. Such parameters include the number of snapshots and basis vectors used in the POD of the heat transfer problem and the number of Ritz modes included in the structural dynamics solution. Additionally the ROMs will be created offline such that the computational expense of the high fidelity simulations will not affect the reduced order nature of the control-oriented design and analysis. The purpose of this work is to avoid the need to solve a full heat transfer problem and structural dynamics problem at every time step. This work aims to formulate the ROMs such that they do need not to be reconstructed as the heat flux boundary conditions and structural dynamics of the system change over time. Thus, one does not need to return to the detailed finite element models once the ROMs have been created. A schematic of the overall goal of this work is shown in Figure 2. 


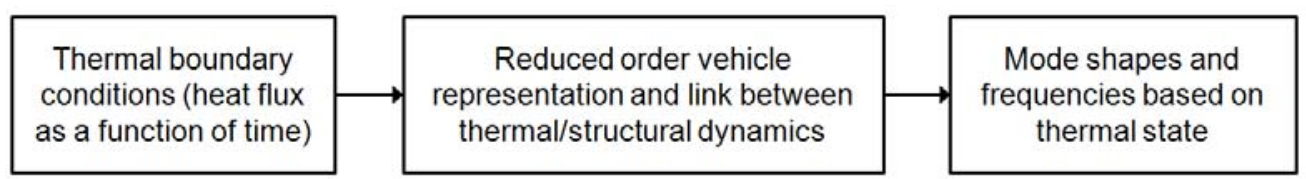

Figure 2. Schematic of fundamental focus of reduced-order modeling.

The result will be a formulation which takes the heat flux boundary conditions as an input and uses a reduced order vehicle representation and link between the thermal and structural disciplines to directly produce vehicle mode shapes and frequencies based on the current thermal state. The following sections describe the various steps to be used in this procedure.

\section{High Fidelity Modeling}

A high-fidelity modeling framework making use of MSC.Nastran thermal and structural finite element analysis has been adopted for the purposes of extracting ROMs and characterizing error. Transient thermal analysis is first run on the model with appropriate heat flux boundary conditions applied to generate nodal temperatures at the time steps of interest. These nodal temperatures are then mapped onto the structural finite element model by creating a spatial field within MSC.Patran. Currently the same grids are being used for both the thermal and structural analyses, but future work will likely make use of different grid resolutions between the models and interpolate the temperatures between the two.

A notional finite element model representative of a hypersonic vehicle control surface has been created for the purpose of this study. The control surface is being studied not just as a representative structure to illustrate the methodology, but because it is believed to have potentially the greatest impact on the overall controllability of the vehicle. Analysis of the transient structural dynamics over the flight profile will provide insight into the effectiveness of the control surface and its impact on the overall vehicle dynamics. A schematic of the envisioned two-dimensional geometry ${ }^{5}$ of the hypersonic vehicle is shown in Fig. 3 with the canard and elevon control surfaces highlighted.

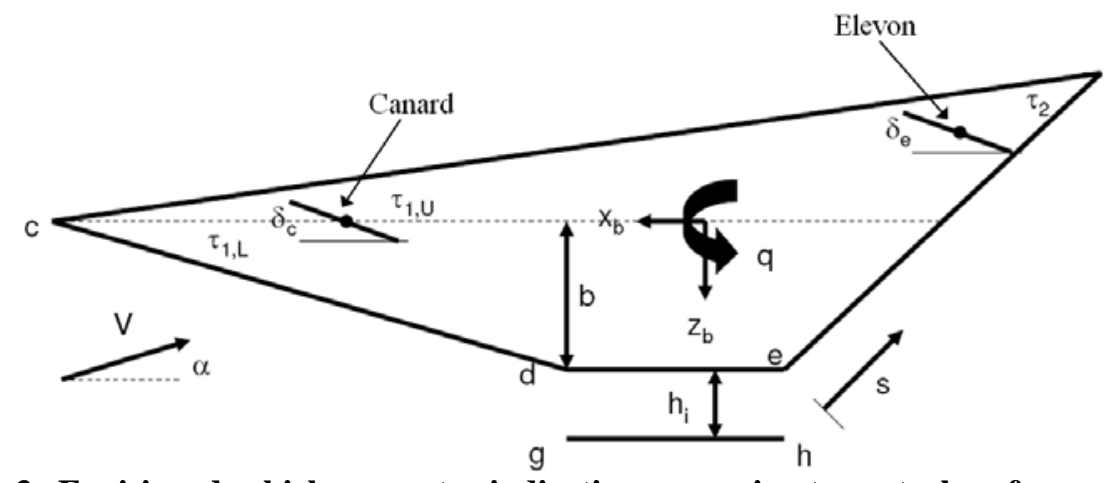

Figure 3. Envisioned vehicle geometry indicating approximate control surface positions. ${ }^{5}$

The control surface model created for this study to represent one of the above control surfaces is shown in Fig. 4 along with an artist rendition of a hypersonic vehicle concept and a finite element model of the NASA X-43A control surface. The control surface is discretized using two-dimensional isoparametric triangular elements in both the thermal and structural finite element models. The overall dimensions and rib pattern have been estimated based on relevant papers ${ }^{5-7}$ are given in Fig. 5 . 


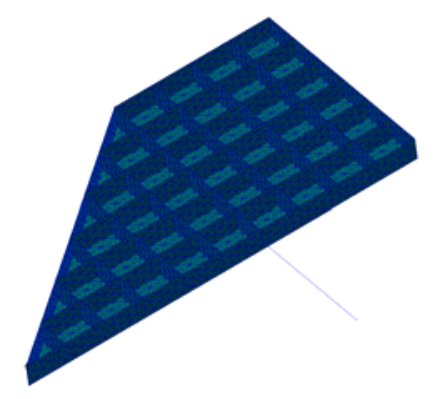

(a)

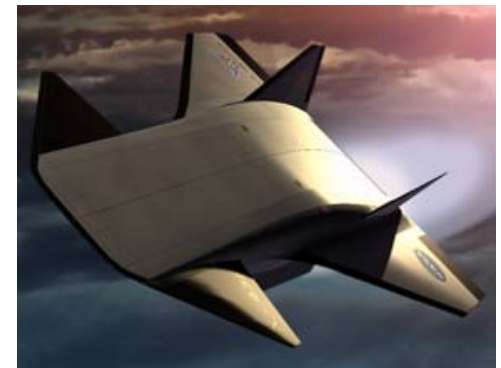

(b)

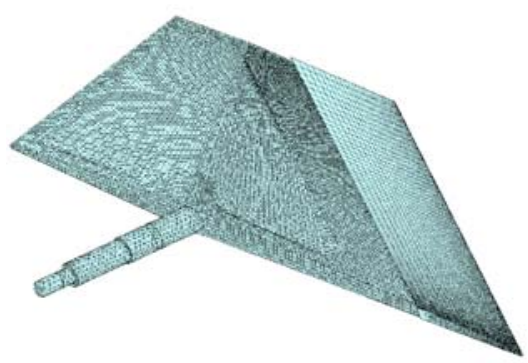

(c)

Figure 4. (a) Control surface model, (b) Rendition of NASA X-43B ${ }^{7}$, (c) FE Model of X-43A control surface. ${ }^{2}$

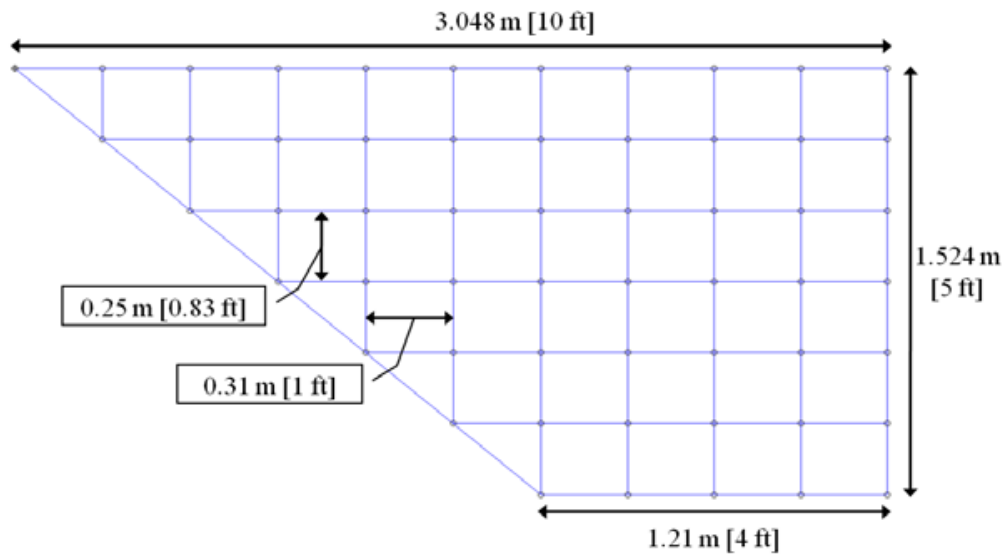

Figure 5. Control surface geometry and dimensions.

The material for the structure was chosen to be PM 2000 due to its high strength at high temperatures and relatively low thermal diffusivity constant. ${ }^{8,9}$ Note that this is a preliminary selection, and that further sizing and investigation will provide more insight into the materials, dimensions, and stacking necessary for a hypersonic control surface application of this type. The thermo-mechanical material properties as used in this study are shown in Table 1. From the given properties, a temperature-dependent Young's Modulus curve was obtained (Fig. 6). For the purpose of this study, Young's Modulus was extrapolated beyond the data given, as material property data spanning the range of temperatures expected in hypersonic applications proves difficult to obtain. All element thicknesses were chosen to be 0.25 inches for this initial study.

Table 1. Thermo-mechanical material properties for PM 2000. ${ }^{8,9}$

\begin{tabular}{|c|c|}
\hline Thermal Conductivity, $k$ & $28 \mathrm{~W} /(\mathrm{m}-\mathrm{K})$ \\
\hline Specific Heat, $c_{p}$ & $740 \mathrm{~J} /(\mathrm{kg}-\mathrm{K})$ \\
\hline Density, $\rho$ & $7180 \mathrm{~kg} / \mathrm{m}^{3}$ \\
\hline Poisson's Ratio, $v$ & 0.33 \\
\hline Young's Modulus, $E$, at $T=193 \mathrm{~K}$ & $215 \mathrm{GPa}$ \\
\hline Young's Modulus, $E$, at $T=473 \mathrm{~K}$ & $205 \mathrm{GPa}$ \\
\hline Young's Modulus, $E$, at $T=673 \mathrm{~K}$ & $185 \mathrm{GPa}$ \\
\hline Young's Modulus, $E$, at $T=873 \mathrm{~K}$ & $172 \mathrm{GPa}$ \\
\hline Young's Modulus, $E$, at $T=973 \mathrm{~K}$ & $160 \mathrm{GPa}$ \\
\hline Young's Modulus, $E$, at $T=1073 \mathrm{~K}$ & $155 \mathrm{GPa}$ \\
\hline Young's Modulus, $E$, at $T=1223 \mathrm{~K}$ & $140 \mathrm{GPa}$ \\
\hline
\end{tabular}




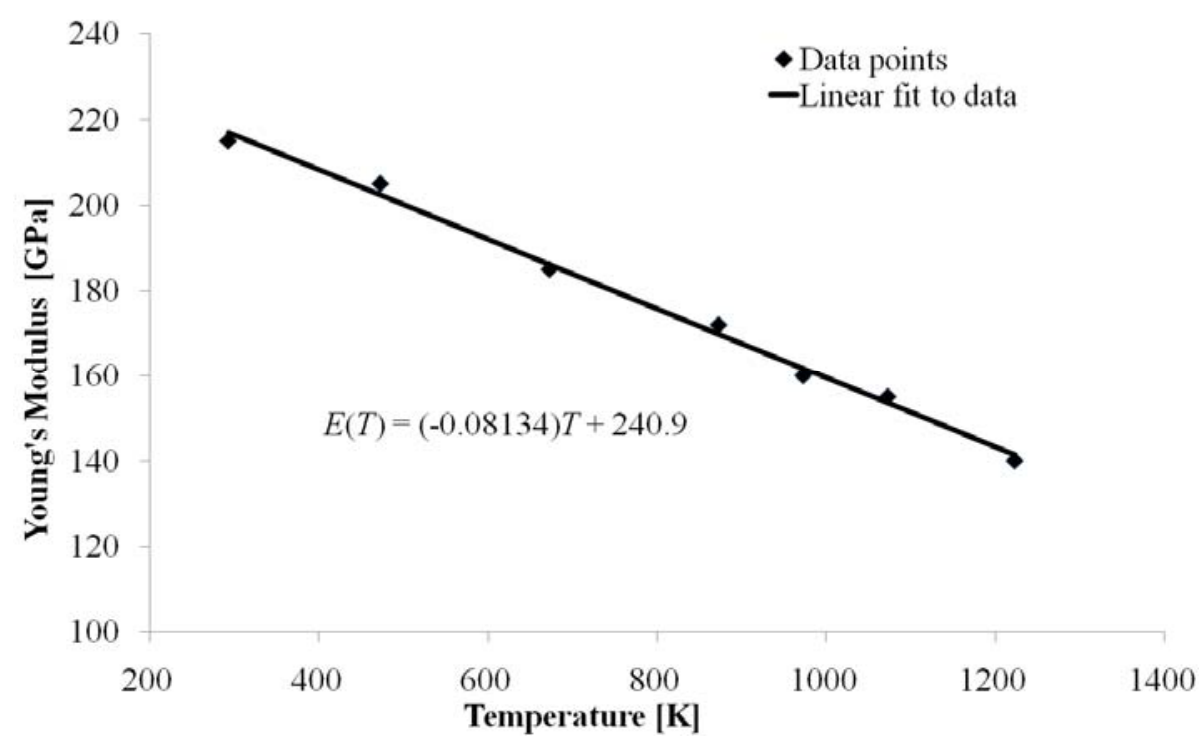

Figure 6. Linear fit used for temperature dependent Young's Modulus of PM 2000 in the finite element model.

\section{Reduced-Order Thermal Modeling: Proper Orthogonal Decomposition}

Proper orthogonal decomposition (POD) is proposed for solution of the transient heat transfer problem. ${ }^{10}$ This allows for determination of the temperature distribution at any time simply by finding a vector of time-dependent coefficients which are the modal amplitudes of the POD basis vectors. The use of such a modal expansion allows one to avoid the need to solve the full-order heat transfer problem at ever time step. The POD basis is optimal in the sense that the energy is concentrated in the lower modes as much as possible so as to allow for truncation of the basis without significant loss of accuracy. The ability to truncate the basis will ultimately mean that the number of time-dependent modal amplitudes to be found will be much less than the number of nodal degrees of freedom in the thermal finite element model. Therefore POD will provide a significant computational advantage in permitting solution through finding modal amplitudes of basis vectors as opposed to a full order vector of nodal temperatures. Once the POD basis is found, there is no need to return to the detailed model and perform full-order simulations. The role of POD in the overall solution process is shown in Fig. 7 with the grey indicating steps that will be bypassed through its use.

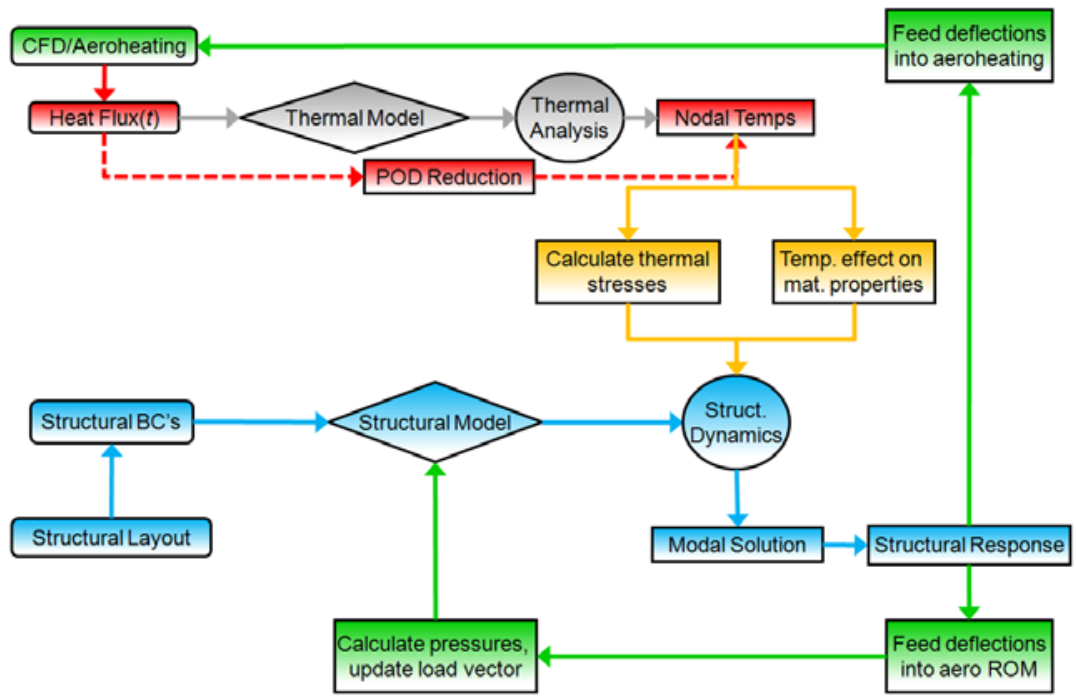

Figure 7. Role of POD in overall solution process. 


\section{III.1Thermal POD Formulation}

The approach makes use of the method of snapshots in determining the POD basis vectors. The formulation begins by generating $N_{s}$ snapshots which are defined as state solutions evaluated at several values of one of more parameters of interest. In the case of the transient heat conduction problem, the snapshots are vectors of nodal temperatures at various time instants. The goal of the POD formulation is to find a reduced basis, $\varphi(x, y, z)$, and time-dependent coefficients, $c(t)$, such that the temperature distribution at any time $t$ can be written as

$$
\left\{\begin{array}{c}
T_{1} \\
\vdots \\
T_{m}
\end{array}\right\}=c_{1}(t)\left\{\begin{array}{c}
\varphi_{1}^{(1)} \\
\vdots \\
\varphi_{m}^{(1)}
\end{array}\right\}+c_{2}(t)\left\{\begin{array}{c}
\varphi_{1}^{(2)} \\
\vdots \\
\varphi_{m}^{(2)}
\end{array}\right\}+\ldots+c_{n}(t)\left\{\begin{array}{c}
\varphi_{1}^{(n)} \\
\vdots \\
\varphi_{m}^{(n)}
\end{array}\right\},
$$

where $T$ refers to the value of the nodal temperature, $m$ is the total number of degrees of freedom in the thermal finite element model, and $n$ is the total number of POD basis vectors retained after truncation. After $N_{s}$ snapshots are generated, the vector of initial temperatures, $T_{0}$, is subtracted from each snapshot. They are then used to form the snapshot matrix, $A$, where each snapshot is a column of it, as

$$
A=\left[\begin{array}{cccc}
T_{1}^{(1)} & T_{1}^{(2)} & \cdots & T_{1}^{\left(N_{s}\right)} \\
T_{2}^{(1)} & T_{2}^{(2)} & \cdots & T_{2}^{\left(N_{s}\right)} \\
\vdots & \vdots & \vdots & \vdots \\
T_{m}{ }^{(1)} & T_{m}^{(2)} & \cdots & T_{m}{ }^{\left(N_{s}\right)}
\end{array}\right] .
$$

As the snapshots will inherently be correlated, we can eliminate redundant information. This is accomplished via the calculation of the correlation matrix, $C$, whose entries are given by integrating over the inner product of the $i$-th and $j$-th columns of the snapshot matrix as

$$
C_{i j}=\frac{1}{N_{s}} \int_{V} A^{(i)^{T}} A^{(j)} d V,
$$

where ()$^{(i)}$ indicates the $i$-th column of the matrix ( ). Because the snapshots are taken from a finite element solution, we have a continuous representation of temperatures over the structure as opposed to strictly discrete nodal values. We can take advantage of this fact in evaluating the above integrals by expanding the nodal temperatures in terms of the shape functions and performing a continuous integral as opposed to a discrete summation. Consider the $3 \times 1$ vector of nodal temperatures for each element in matrix form as

$$
\mathcal{T}(x, y, z, t)=N T_{e}
$$

where $T_{e}$ is the vector of elemental temperatures for a specific element and $N$ is the finite element shape function matrix. For this study, it is simply the row vector of standard finite element shape functions for the isoparametric triangular element. Moreover, consider the transformation matrix, $T^{e G}$, that relates local element degrees of freedom for element $e$ with global ones, i.e., $T_{e}=T^{e G} T$, so that Eq. (4) becomes

$$
\mathcal{T}(x, y, z, t)=N T^{e G} T
$$

Using Eq. (4) in Eq. (3), the expression for the elements of the correlation matrix becomes 


$$
C_{i j}=\frac{1}{N_{s}} \sum_{e}\left[\int_{V} T^{(i)^{T}} T^{e G^{T}}\left(N^{T} N d V\right) T^{e G} T^{(j)}\right]
$$

where the summation indicates that the integral is summed over all elements for each entry in the correlation matrix. Again, the $i$ and $j$ indices refer to the snapshot numbers. Making use of the isoparametric map, we can express the integral over the shape functions in terms of the isoparametric coordinates, $\xi$ and $\eta$, the element thickness, $t$, and the determinant of the Jacobian resulting from the isoparametric coordinate transformation, $J$, as

$$
\int_{V} N^{T} N d V=t \int_{0}^{1} \int_{0}^{1-\xi}\left(\left\{\begin{array}{l}
N_{1} \\
N_{2} \\
N_{3}
\end{array}\right\}\left[\begin{array}{lll}
N_{1} & N_{2} & N_{3}
\end{array}\right] \operatorname{det} J\right) d \eta d \xi
$$

Now, the eigenvalues, $\lambda$, and eigenvectors, $v$, of the correlation matrix are then used along with the columns of the snapshot matrix to calculate the POD vectors, $\varphi$, i.e.,

$$
\varphi_{k}=\frac{1}{\sqrt{N_{s} \lambda_{k}}} \sum_{i=1}^{N_{s}} v_{i}^{(k)} A^{(i)},
$$

where $v_{i}^{(k)}$ is the $i$-th element of the $k$-th eigenvector. This produces the orthonormal basis that will be used to construct the temperature distribution at any time instant of interest. As mentioned above, the optimality of the POD basis is a result of the correlation of the snapshots, which leads to a rapid decay of the eigenvalues of the correlation matrix. POD modes whose corresponding eigenvalue is of small magnitude do not contribute significantly to the solution, and thus the corresponding POD mode can be eliminated from the set. The plot in Fig. 8 shows an example of the magnitudes of the eigenvalues of the correlation matrix generated from the control surface model containing 4,056 degrees of freedom with 11 snapshots taken.

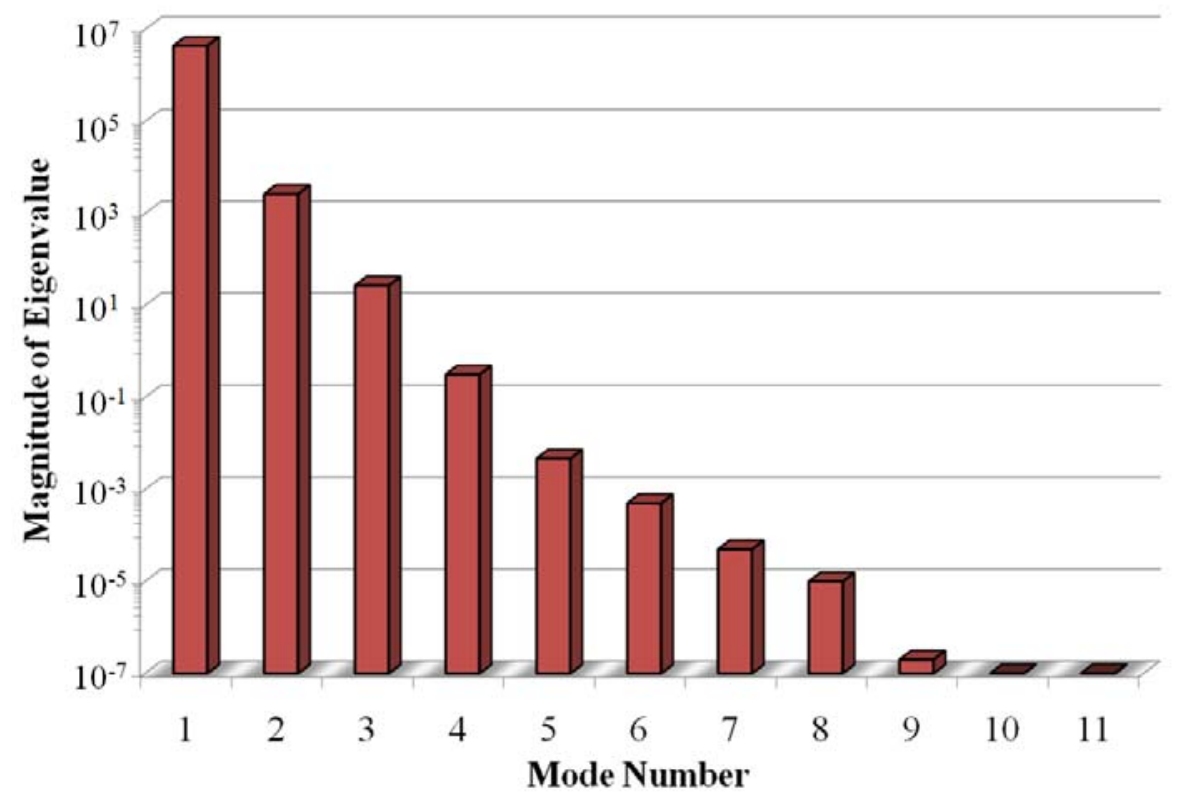

Figure 8. Eigenvalues of correlation matrix for 11 snapshot case applied to control surface model.

Once the POD basis vectors are found and the basis is truncated accordingly, it remains to calculate the timedependent modal amplitudes at the time steps of interest. This work makes use of the methodology developed in 
Ref. 10. An overview of the procedure will be given here, and the interested reader is referred to the referenced paper for further detail. The process begins by forming the modal matrix $\Phi$ whose columns are the POD basis vectors. Note that at this stage we will assume that the POD basis has been truncated based on the eigenvalue analysis as described above. Consider the thermal finite element system of equations given by

$$
M \dot{T}+K T=f
$$

where $M$ is the lumped form of the thermal capacitance matrix and $K$ is the thermal conductivity matrix. Here it is assumed that the Eq. (9) has been recast to account for the initial conditions being subtracted from the vector of nodal temperatures as mentioned in the discussion of the snapshot matrix. The system of equations is transformed to make all of the entries in the diagonal matrix $M$ unity, thus reducing it to the identity matrix. This is accomplished using the matrix $M^{-1 / 2}$ to transform $T$ so

$$
T=M^{-1 / 2} T^{*} .
$$

Under this transformation, the system of equations becomes of the form

$$
\dot{T}^{*}+K^{*} T^{*}=f^{*} .
$$

From the modal expansion as given in Eq. (1), we can express the vector of nodal temperatures as a matrix-vector product of the form

$$
\left\{\begin{array}{c}
T_{1} \\
\vdots \\
T_{m}
\end{array}\right\}=\left[\begin{array}{cccc}
\varphi_{1}^{(1)} & \varphi_{1}^{(2)} & \ldots & \varphi_{1}^{(l)} \\
\varphi_{2}^{(1)} & \varphi_{2}^{(2)} & \ldots & \varphi_{2}^{(l)} \\
\vdots & \vdots & \ldots & \vdots \\
\varphi_{m}^{(1)} & \varphi_{m}^{(2)} & \ldots & \varphi_{m}^{(l)}
\end{array}\right]\left\{\begin{array}{c}
c_{1}(t) \\
\vdots \\
c_{n}(t)
\end{array}\right\}=\Phi c
$$

where each column of the modal matrix is one of the POD basis vectors. The same transformation as used in Eq. (10) is then applied to the temperatures in Eq. (12) to transform from $T$ to $T^{*}$. From this, we can substitute for $T^{*}$ into Eq. (11) to express it in terms of the modal matrix and the modal amplitudes, $c$. Pre-multiplying the resulting system by $\Phi^{T}$, we get

$$
\Phi^{T} \Phi \dot{c}+\Phi^{T} K^{*} \Phi c=\Phi^{T} f^{*}
$$

At this stage, the equations are reduced from a $m$-dimensional system to a $n$-dimensional one. Because the POD basis vectors constitute an orthonormal set, the coefficient matrix of the time-derivatives of $c$ reduces to the identity matrix. However, since the POD basis vectors are not eigenmodes of the original problem, they do not diagonalize the transformed thermal conductivity matrix $K^{*}$. Due to this fact, one further step is taken to diagonalize the transformed thermal conductivity matrix by using the matrix of eigenvectors, $W$, and applying it to the whole system, thus decoupling the equations. Following this diagonalization, the system of equations will finally become

$$
\hat{\dot{c}}+\Gamma \hat{c}=\hat{f}
$$

where $\Gamma$ is a diagonal matrix containing the eigenvalues of the transformed thermal conductivity matrix $K^{*}$. As Eq. (14) is now decoupled, we can express the $i$-th equation as

$$
\hat{\dot{c}}_{i}+\gamma_{i} \hat{c}_{i}=\hat{f}_{i}, i=1,2 \ldots, n
$$

with $\gamma_{i}$ being the $i$-th eigenvalue of the transformed thermal conductivity matrix $K^{*}$. The advantage of decoupling the equations is that we can now solve the equations analytically such that a numerical time-stepping scheme is not 
needed and the solution at any time instant of interest can immediately be generated. The analytical solution to Eq. (15) is given by

$$
\hat{c}_{i}(t)=\hat{f}_{i} \frac{1-\mathrm{e}^{-\gamma_{i} t}}{\gamma_{i}}
$$

Once one solves for the transformed vector of modal amplitudes at the time instant of interest, the temperature distribution can be calculated by reversing the series of variable transformations and using the modal expansion in Eq. (12) to find the nodal temperatures, i.e.,

$$
\mathcal{T}(x, y, z, t)=R \hat{c}(t)+T_{0}
$$

with $R=M^{1 / 2} \Phi W$. Note that prior to the first transformation, the initial condition was subtracted from the temperature vector, as done in Ref. 10, to make the initial condition a uniform zero temperature. For this reason the vector of initial temperatures must be added back to the solution in Eq. (17). Figure 9 gives an overview of the equation transformations leading from the full, coupled system of thermal finite element equations to the reduced, uncoupled set of ordinary differential equations for the time-dependent modal amplitudes (as proposed in Ref. 10).

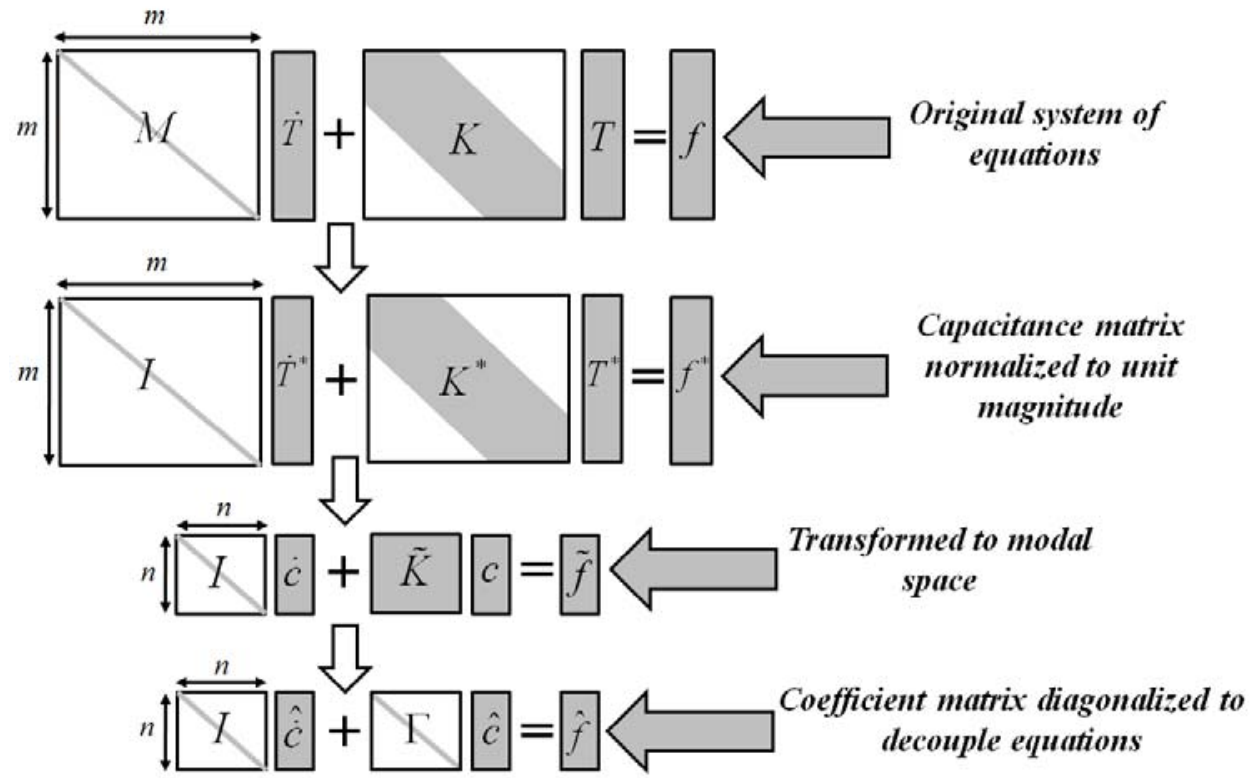

Figure 9. Sequence of equation transformations leading to reduced order decoupled system. ${ }^{10}$

\section{III.2Thermal POD Example}

The POD methodology was applied to the control surface finite element model and a time interval from 0-500 seconds was considered. A simple uniform heat flux of $10 \mathrm{~W} / \mathrm{cm}^{2}$ was applied to the top skin with all other surfaces having a zero applied heat flux. As work is currently being undertaken to fully automate the snapshot procedure, 26 snapshots were taken from a high-fidelity transient thermal solution using Nastran in evenly space intervals of 20 seconds. It is expected that a greater number of snapshots will be needed in the future in order to attain the desired accuracy. Figure 10 shows an example temperature contour plot at 300 seconds. 


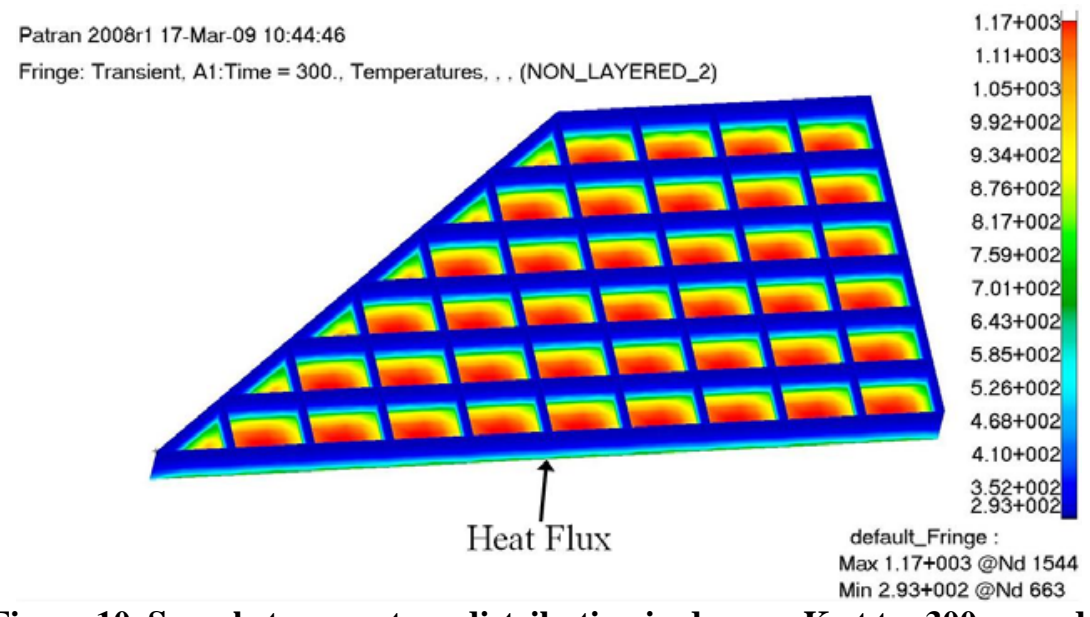

Figure 10. Sample temperature distribution in degrees $K$ at $t=\mathbf{3 0 0}$ seconds.

The basis was truncated significantly from the original finite element model containing 4,056 degrees of freedom and only four POD modes were included in the solution. Figure 11 shows the first 10 eigenvalues of the correlation matrix for the 26 snapshot case. All eigenvalues corresponding to modes $11-26$ were approximately zero. To analyze the performance of the POD methodology, the temperatures were calculated at various time instants throughout the transient. An error vector was calculated by subtracting the vector of nodal temperatures obtained by POD from that obtained from a high-fidelity simulation and taking its absolute value. The error is quantified by taking $\|$ Error $\|_{\infty}$ and $\|$ Error $\|_{2}$ as shown in Table 2. The percentage error vector for each time instant is calculated by taking the difference between the POD and FEA temperature vectors and dividing each entry in the resulting vector by the value of the corresponding nodal temperature from FEA. This relative error is quantified by taking $\| \%$ Error $\|_{\infty}$ and $\| \%$ Error $\|_{2}$ as also shown in the Table 2.

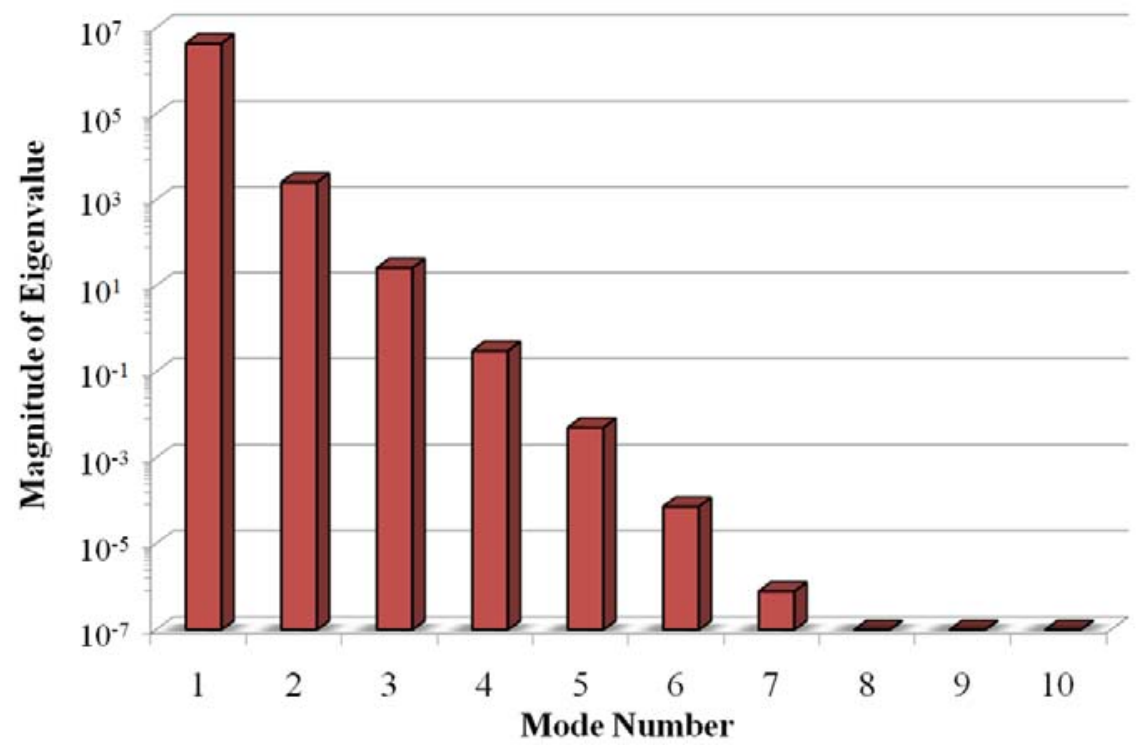

Figure 11. First 10 eigenvalues of the correlation matrix for 26 snapshot case. 
Table 2. Norms of POD error at selected time instants.

\begin{tabular}{|c|c|c|c|c|}
\hline Time $[\mathrm{s}]$ & $\|$ Error $\|_{\infty}[\mathrm{K}]$ & $\|$ Error $\|_{2}[\mathrm{~K}]$ & $\| \%$ Error $\|_{\infty}[\%]$ & $\| \%$ Error $\|_{2}[\%]$ \\
\hline 40 & 2.1 & 53.6 & 0.7 & 17.6 \\
\hline 80 & 4.7 & 122.7 & 1.5 & 38.5 \\
\hline 120 & 7.6 & 189.6 & 2.3 & 57.0 \\
\hline 160 & 9.6 & 247.5 & 2.9 & 71.9 \\
\hline 200 & 11.7 & 298.3 & 3.3 & 94.3 \\
\hline 240 & 13.9 & 346.5 & 3.8 & 107.8 \\
\hline 280 & 16.1 & 396.2 & 4.1 & 120.5 \\
\hline 320 & 18.4 & 450.4 & 4.4 & 134.3 \\
\hline 360 & 20.9 & 510.7 & 4.7 & 149.0 \\
\hline 400 & 23.5 & 577.9 & 5.4 & 164.4 \\
\hline 440 & 26.2 & 652.1 & 6.1 & 180.2 \\
\hline 480 & 29.0 & 733.1 & 6.7 & \\
\hline
\end{tabular}

To provide an indication of the spatial distribution of the error, nodes were numbered according to their location within the finite element model and the absolute value of the percentage error was plotted as a function of node index as in Fig. 12. As can be seen, the highest relative error generally occurs in the interior ribs. This is expected as this is where the greatest temperature gradients occur. Further investigation into reducing this error by varying the number of snapshots and snapshot spacing is planned for the future.

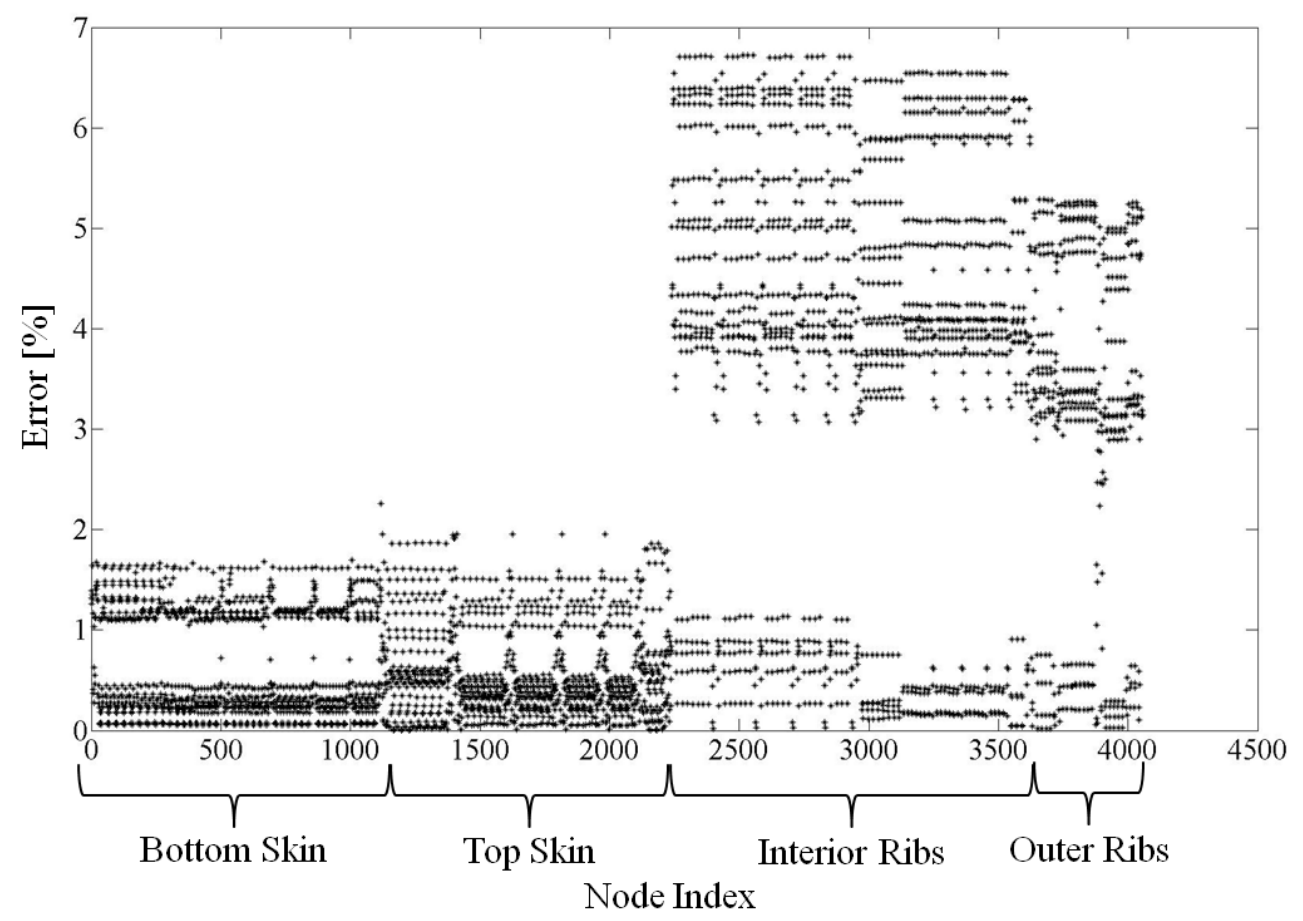

Figure 12. Percentage error vs. node index at $t=480$ seconds.

\section{III.3Time-Varying Heat Flux Boundary Conditions}

Note that results up to this point have been taken from simulations containing a heat flux that is constant in time. The overall goal of this work is to be able to take in a time-dependent heat flux at the vehicle surface and directly generate the structural mode shapes and frequencies without having to solve a high-fidelity thermal and structural problem. It thus becomes necessary to extend the above POD methodology so that it can incorporate the effect of transient heat flux boundary conditions at the surface without reconstructing the ROM. To do so, it is proposed to follow the methodology used in Ref. 10 for dealing with time-dependent heat loads. The method takes advantage of the fact that the original POD basis vectors do not need to be updated as the heat flux changes. This is due to the fact that the basis vectors are taken to be admissible functions, which need only to satisfy the essential boundary 
conditions. In the thermal case essential boundary conditions are fixed temperature boundary conditions. Because this model only contains natural boundary conditions (heat flux boundary conditions), the POD basis vectors generated from the initial snapshots do not need to be regenerated when the heat flux changes because they will always satisfy the essential boundary conditions. Therefore, once the POD basis vectors are generated, no additional finite element runs are necessary as the heat flux changes. Begin by assuming that one can represent the thermal load vector as the multiplication of a time-dependent scalar function, $h$, and a spatially varying load vector, $f$, such that the system of equations becomes

$$
M \dot{T}+K T=h(t) f(x, y, z)
$$

After decoupling the equations according to the transformations described previously, each equation will be of the form,

$$
\hat{\dot{c}}_{i}+\gamma_{i} \hat{c}_{i}=h(t) \hat{f}_{i},
$$

and the analytical solution to each equation can be expressed as

$$
\hat{c}_{i}(t)=\hat{f}_{i} \mathrm{e}^{-\gamma_{i} t} \int_{0}^{t} \mathrm{e}^{\gamma_{i} t^{\prime}} h\left(t^{\prime}\right) d t^{\prime} .
$$

Note that solution of the equations requires integration of the time-dependent scalar function from initial time to final time. It is unlikely that the thermal load vector will be able to be expressed as a single time-dependent function multiplied by a single spatially varying vector. It is therefore expected that a modal expansion will need to be performed on the thermal load vector in order to separate the temporal and spatial dependence of the thermal load. After such an expansion, the system of thermal finite element equations becomes

$$
M \dot{T}+K T=\sum_{l=1}^{L} h_{l}(t) f_{l}
$$

The system can then be diagonalized as described previously and solved analytically for the response. As described in Ref. 10, each term on the right-hand side of the above equation can be treated individually and solved for each corresponding modal amplitude using

$$
\hat{c}_{l, i}(t)=\hat{f}_{l, i} \mathrm{e}^{-\gamma_{i} t} \int_{0}^{t} \mathrm{e}^{\gamma_{i} t^{\prime}} h_{l}\left(t^{\prime}\right) d t^{\prime}
$$

and the complete solution can be obtained as

$$
\hat{c}=\sum_{l=1}^{L} \hat{c}_{l}
$$

The challenge in this work stems from the fact that the temporal variation in surface heat flux will not be known ahead of time as it depends on the structural deformation. Thus it is expected that a modal decomposition strategy similar to the one used for temperature will need to be used to generate the modal expansion for the load vector in Eq. (21). Once the basis vectors $f$ are generated, it remains to calculate the time-dependent amplitudes $h(t)$ at the time instant of interest given the actual heat flux vector calculated from the aeroheating analysis. Additionally, the value of the heat flux will be sampled at discrete time instants along the flight trajectory, with the sampling rate to be determined. Since only a discrete set of coefficients $h$ will be known along the transient, some method for approximating a functional representation of the coefficients in between the discrete points will be necessary to perform the integral in Eq. (22). Methods for implementing such strategies are currently being investigated by the authors. 


\section{Formulation of Structural Dynamics Under Thermal Loads}

As discussed in the introduction, knowledge of the structural dynamics of a hypersonic vehicle over time is necessary as changing natural frequencies and mode shapes will result in migration of the poles and zeros of the linearized aircraft dynamics which determine the required robustness of the control system. ${ }^{2}$ The problem is further complicated by the fact that control and design models require a structural dynamics solution that possesses a relatively low number of states. For these reasons it becomes necessary to determine the structural dynamics of the vehicle over time while retaining only the essential physics. To achieve this goal, a methodology for determination of mode shapes and frequencies as a function of temperature in a reduced sense is proposed. This approach will make use of the aforementioned POD solution to first determine the thermal state of the structure at various time instants along a flight trajectory. The temperature distribution must then be used in conjunction with already known functional representations of temperature-dependent material properties to modify the equations of motion accordingly and solve for the mode shapes and frequencies of structural components.

\section{IV.1 Modified Modal Formulation}

Because transient heating will modify the stiffness matrix when a variable Young's Modulus and thermal stresses are considered, the full structural dynamics eigenvalue problem would need to be solved at every time step in a high-fidelity analysis. This work aims to decrease the computational burden and number of states by reducing the order of the eigenvalue solution. The methodology involves use of the discrete form of the Ritz method with the modal matrix determined by finding the free vibration mode shapes at a reference thermal state representing some average temperature distribution. This technique takes advantage of the fact that the Ritz modes need only to satisfy the geometric boundary conditions. ${ }^{12}$ Because the reference modes will always satisfy these boundary conditions, they are applicable to use throughout the transient and need not to be updated. Therefore, once the reference modes are found, solution of the full-order structural dynamics problem is no longer required. The equations of motion can then be transformed to modal space using the modal matrix assembled from the reference free vibration modes as

$$
\Phi_{r e f}^{T} m \Phi_{r e f} \ddot{\eta}+\Phi_{r e f}^{T} k^{*}(T) \Phi_{r e f} \eta=\Phi_{r e f}^{T} f,
$$

where $\Phi_{r e f}$ is the modal matrix obtained by finding the free vibration mode shapes at a reference thermal state and normalizing them with respect to the mass matrix. The modified stiffness matrix, $k^{*}$, is given by

$$
k^{*}(T) \equiv k(T)+k_{G}(T),
$$

where $k(T)$ is the conventional stiffness matrix and $k_{\mathrm{G}}(T)$ is the geometric stiffness matrix due to thermal stresses. As described in the introduction, the geometric stiffness matrix will be calculated by solving a static finite element problem based on the thermal loads from the temperatures at the current time step and coefficients of thermal expansion. The number of modal degrees of freedom, $\eta$, is reduced by strategically truncating the modal basis. If the Young's Modulus were constant, the stiffness matrix would be diagonalized by the modal matrix, thus decoupling the system of differential equations. However, by including the effect of a temperature-variable Young's Modulus, the stiffness matrix will be changing over time due to transient heating. The global stiffness matrix will therefore no longer be diagonalized by the modal matrix and the system becomes coupled. Initially, the free vibration problem will be considered and natural frequencies and mode shapes will be found.

\section{IV.2 Coupled Thermo-Elastic Example}

To exemplify the methodology and the effect of transient heating on the structural equations of motion, a onedimensional case is considered. For the thermal part of the problem, a one-dimensional heat conduction problem with two different heat fluxes applied at the ends was analyzed as shown in Fig. 13. The beam has a one meter by one meter cross section and is 60 meters long. The temperatures were calculated every 50 seconds in the interval from $0-200$ seconds and are given in Fig. 14. 


$$
\begin{aligned}
& q=1000 \mathrm{~W} / \mathrm{m}^{2} \longrightarrow \square \longleftarrow q=500 \mathrm{~W} / \mathrm{m}^{2} \\
& \text { Initial Condition: } T(x, 0)=293 \mathrm{~K} \\
& \text { (Uniform Temperature) }
\end{aligned}
$$

Figure 13. Boundary conditions and initial conditions for one-dimensional thermal problem.

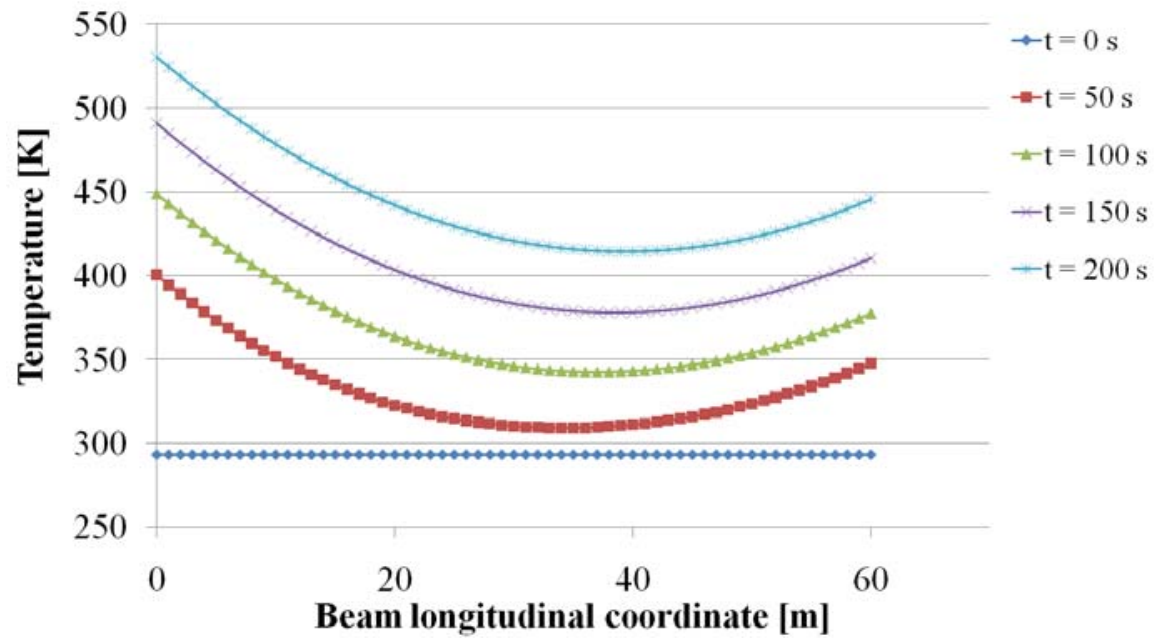

Figure 14. Temperature distributions at selected time instants from thermal FEA.

The nodal temperatures shown in Fig. 14 were then mapped onto a structural cantilever beam finite element model with the left end $(x=0)$ clamped. The grids used for the thermal and structural models were the same. As the structural model contained a temperature varying Young's Modulus, the applied thermal loading modified the stiffness of the structure. The material was taken to be Titanium and the temperature dependence of Young's Modulus was extrapolated based on data from Vosteen. ${ }^{13}$ The approximate linear representation for Young's Modulus used in the finite element model is given in Fig. 15.

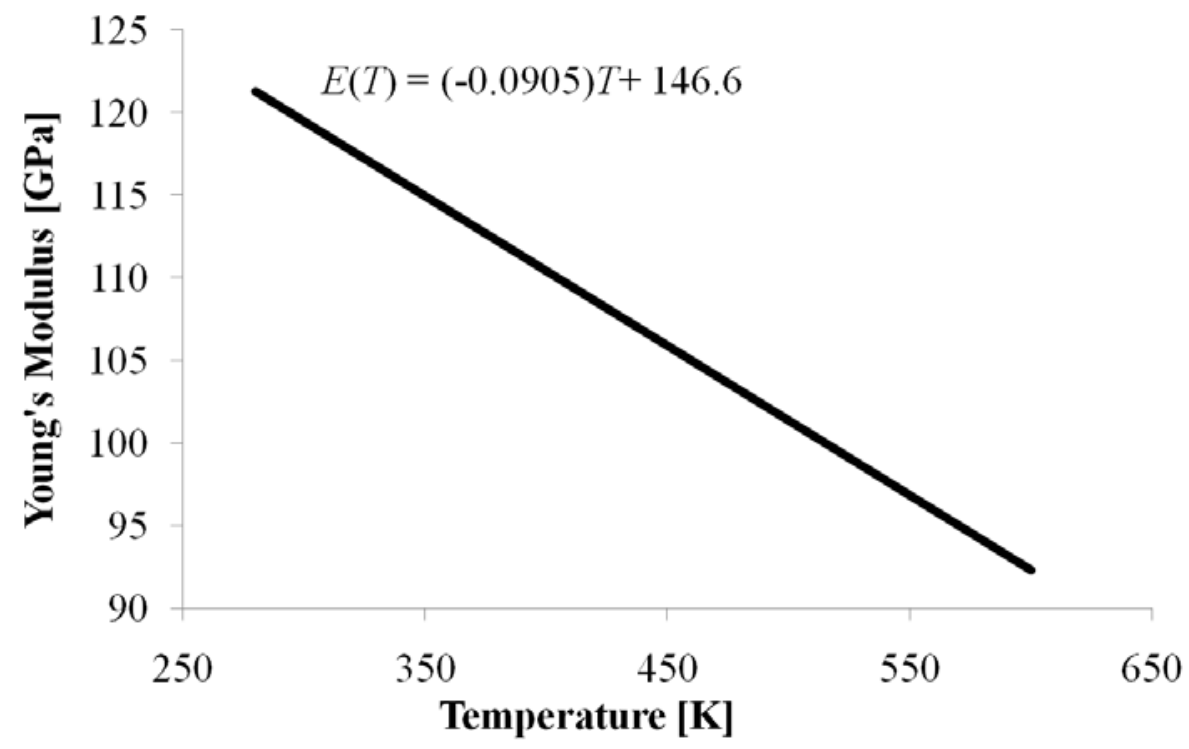

Figure 15. Dependence of Titanium's Young's Modulus with temperature for one-dimensional case study.

A thermal load case was created at 50-s intervals and Nastran analysis was run with a DMAP (Direct Matrix Abstraction Program) alter in the input file requesting output of the structural stiffness matrix for each thermal load 
case. The stiffness matrix generated at each time instant was then used to calculate the generalized stiffness matrix in order to assess its diagonality and thus the amount of coupling in the equations of motion. For each 50-s interval, the generalized stiffness matrix was calculated using

$$
\Phi_{r e f}^{T} k(T) \Phi_{r e f}=\bar{k}(T)_{5 x 5},
$$

where $\Phi_{\text {ref }}$ was assembled using five free vibration modes at the $t=100 \mathrm{~s}$ thermal case. The stiffness matrix $k(T)$ was evaluated using the temperatures at the specific time instant and the functional representation of Young's Modulus from Fig. 15. No thermal stress effects are included in this example. To quantify the diagonality of the matrix, a matrix diagonality ratio was calculated for each row of the $5 \times 5$ matrix as given below

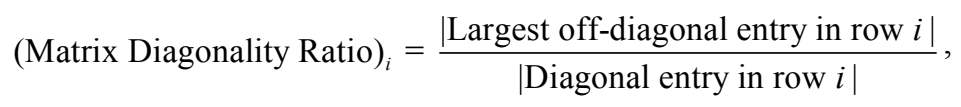

where values closer to zero indicate a matrix that is more diagonally dominant. Results from this case are shown in Table 3.

Table 3. Matrix diagonality ratios for selected time instants.

\begin{tabular}{|c|c|c|c|c|c|c|}
\cline { 3 - 7 } \multicolumn{2}{c|}{} & \multicolumn{5}{|c|}{ Time Step } \\
\cline { 3 - 7 } \multicolumn{2}{c|}{} & $\boldsymbol{t}=\mathbf{0 ~ s}$ & $t=\mathbf{5 0 \mathrm { s }}$ & $\boldsymbol{t}=\mathbf{1 0 0 \mathrm { s }}$ & $\boldsymbol{t}=\mathbf{1 5 0 \mathrm { s }}$ & $\boldsymbol{t}=\mathbf{2 0 0 \mathrm { s }}$ \\
\hline \multirow{4}{*}{$\begin{array}{c}\text { Matrix } \\
\text { Diagonality } \\
\text { Ratio }\end{array}$} & Row 1 & $1.785 \times 10^{-1}$ & $2.466 \times 10^{-2}$ & $1.561 \times 10^{-4}$ & $1.324 \times 10^{-2}$ & $2.267 \times 10^{-2}$ \\
\cline { 2 - 7 } & Row 2 & $6.128 \times 10^{-2}$ & $1.177 \times 10^{-2}$ & $4.700 \times 10^{-6}$ & $6.622 \times 10^{-3}$ & $1.096 \times 10^{-2}$ \\
\cline { 2 - 7 } & Row 3 & $3.573 \times 10^{-2}$ & $7.793 \times 10^{-3}$ & $5.999 \times 10^{-7}$ & $4.446 \times 10^{-3}$ & $7.280 \times 10^{-3}$ \\
\cline { 2 - 7 } & Row 4 & $2.135 \times 10^{-2}$ & $6.398 \times 10^{-3}$ & $1.239 \times 10^{-7}$ & $3.648 \times 10^{-3}$ & $5.933 \times 10^{-3}$ \\
\cline { 2 - 7 } & Row 5 & $7.850 \times 10^{-3}$ & $2.352 \times 10^{-3}$ & $2.113 \times 10^{-8}$ & $1.341 \times 10^{-3}$ & $2.180 \times 10^{-3}$ \\
\hline
\end{tabular}

As seen from Table 3, the matrix becomes more diagonal in moving from $t=0 \mathrm{~s}$ to the reference case at $t=100 \mathrm{~s}$ and becomes less diagonal as the modes depart from the reference case in moving from $t=100 \mathrm{~s}$ to $t=200 \mathrm{~s}$. In the free vibration case, the coupling of the equations is irrelevant as the equations of motion reduce to an eigenvalue problem due to orthogonality with respect to mass, that is, for $f=0$, one has

$$
\left(\Phi_{r e f}^{T} k^{*}(T) \Phi_{r e f}-\omega^{2} I\right) \eta=0 .
$$

However, the failure of the generalized stiffness to be diagonalized in the case of forced vibration results in the following coupled system of ordinary differential equations

$$
\ddot{\eta}+\Phi_{r e f}^{T} k^{*}(T) \Phi_{r e f} \eta=\Phi_{r e f}^{T} f(t) .
$$

The solution of the forced vibration problem can be obtained using a direct numerical integration appropriate to the form in Eq. (29).

\section{Overall Computational Framework}

Based on the integrated thermo-elastic solution methodology described in this work, a computational framework has been developed to implement the method. A flowchart of the framework is shown in Fig. 16. The process begins by performing high-fidelity thermal analysis and generating the nodal temperatures at the time instants of interests. In the process, the thermal capacitance matrix, thermal conductivity matrix, and thermal load vector are written to output files using DMAP. At this point, the snapshot matrix is generated, the POD basis vectors are formed, and the thermal finite element equations are transformed to modal space using the modal matrix. The system of ordinary differential equations is then solved for the time-dependent modal amplitudes at each time step of interest and they 
are used in the modal expansion to calculate the corresponding temperature distribution. The calculated temperatures are then mapped onto the structural model which contains a temperature dependent Young's Modulus. A thermal load case is also generated for each temperature distribution and solution of the static problem is used to calculate the thermal stresses and the geometric stiffness matrix. The reference thermal state is identified and used to generate the reference modes and assemble the modal matrix. A normal modes solution is then executed for each thermal load case in order to generate the equations of motion at each time step. The equations of motion are transformed to modal space at each time step using the reference modes and integrated to find the modal response.

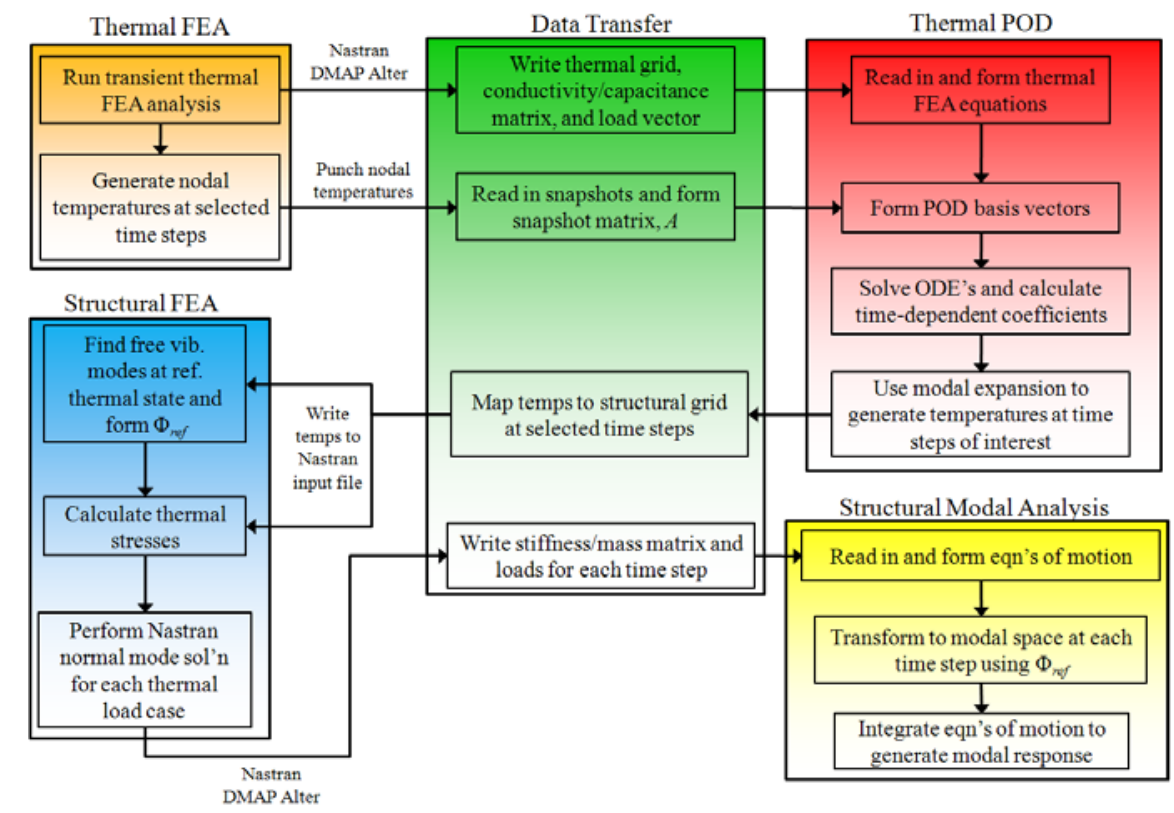

Figure 16. Flowchart of overall computational framework for coupled thermo-elastic solution process.

\section{Concluding Remarks}

This work begins by outlining the challenges associated with hypersonic vehicle design and analysis due to strong interactions among aerodynamics, heat transfer, elastic airframe, propulsion system, flight dynamics, and controls. The specific problem of addressing the impact of aerodynamic heating on vibration mode shapes and frequencies was introduced and the overall high-fidelity solution process is explained. The motivation is associated with the need to reduce the number of states in control system and vehicle design and analysis simulations.

A reduced order, integrated thermo-elastic solution procedure was outlined for the purpose of determining the structural dynamics response over a flight profile. A technique for solving the thermal finite element equations in modal space has been developed by making use of the proper orthogonal decomposition basis vectors extracted from high-fidelity thermal simulations over time. The POD method was applied to a representative control surface model with fixed heat flux boundary conditions and results were compared with those obtained from FEA. An initial comparison of the error at various time steps throughout the transient indicates good accuracy. Additionally, the use of POD enabled reduction of the problem from 4,056 to four degrees of freedom, thus resulting in significant gains in efficiency and reduction of number of states. A technique for extending the current POD formulation to account for transient heat flux boundary conditions sampled from aeroheating analysis at discrete trajectory points was outlined. Future work will implement this technique with the heat flux updated at a rate that is yet to be determined.

A method making use of the discrete form of the Ritz method with mode shapes taken from free vibration modes at a reference thermal state was described for the determination of the structural dynamics over time. This method was applied to a one-dimensional case in which the temperatures are first generated in 50-s intervals and mapped onto the structure. Application of the Ritz method shows coupling of the equations of motion at time instants other than that at which the reference modes are taken. The differences in solving the free and forced vibration problems were discussed. Finally, the overall computational framework as implemented for this work was summarized. Further work will apply this method to a control surface model to determine the frequencies and mode shapes of the structure over time. The effect of the transient structural dynamics on the poles and zeros of the linearized system 
will then be analyzed to assess vehicle controllability. This information will be used to provide insight into the thermo-structural design and material selection with the goal of improving overall flight dynamic performance.

\section{Acknowledgments}

This work was supported by the Michigan-AFRL Collaborative Center in Control Science (MACCCS) under grant number FA 8650-07-2-3744 (Air Force Research Laboratory/Air Vehicles Directorate).

\section{References}

${ }^{1}$ Bolender, M., and Doman, D., "Modeling Unsteady Heating Effects on the Structural Dynamics of a Hypersonic Vehicle," Proceedings of the 2006 AIAA Atmospheric Flight Mechanics Conference, Keystone, Colorado, AIAA 2006-6646.

${ }^{2}$ Lindell, M.C. and Amundsen, R.M., "Nonlinear Thermal/Structural Analysis of Hypersonic Vehicle Hot Structures," Presentation Slides, NASA Workshop on Innovative Finite Element Solutions to Challenging Problems, NASA GSFC, May 2000.

${ }^{3}$ Collier, C.S., "Thermoelastic Formulation of Stiffened, Unsymmetric Composite Panels for Finite Element Analysis of High Speed Aircraft," Proceedings of the $35^{\text {th }}$ Annual AIAA Structures, Structural Dynamics, and Materials Conference, Hilton Head, South Carolina, AIAA 94-1579.

${ }^{4}$ Culler, A., Williams, T., and Bolender, M., "Aerothermal Modeling and Dynamic Analysis of a Hypersonic Vehicle," Proceedings of the 2007 AIAA Atmospheric Flight Mechanics Conference, Hilton Head, South Carolina, AIAA 2007-6395.

${ }^{5}$ Oppenheimer, M., Skujins, T., Bolender., M., and Doman, D., “A Flexible Hypersonic Vehicle Model Developed with Piston Theory," Proceedings of the 2007 AIAA Atmospheric Flight Mechanics Conference, Hilton Head, South Carolina, AIAA2007-6396.

${ }^{6}$ Thomas, J., and Powell, W., "The NASP Program: A Status Report," Proceedings of the 1992 AIAA Space Programs and Technologies Conference, Huntsville, Alabama, AIAA-92-1417.

7“Marshall Space Flight Center News," 5 August 2004, Marshall Space Flight Center, Last Accessed 3/18/2009,

$<$ http://www.msfc.nasa.gov/newsroom/news/photos/2002/2002images/x-43b-sunset2.jpg>.

"'Schwarzkopf Plansee PM 2000," Matweb Material Property Data, Last Accessed 3/18/2009,

$<$ http://www.matweb.com/search/DataSheet.aspx?MatGUID=3601ea51c9214945a63e9330afb457d3\&ckck=1>

${ }^{9}$ Echsler, H., Martinez, E., Singheiser, L., and Quadakkers, W., "Residual Stresses in Alumina Scales Grown on Different Types of Fe-Cr-Al Alloys: Effect of Specimen Geometry and Cooling Rate," Materials Science and Engineering, Vol. 384, No. 1-2, 25 Oct. 2004, pp. 1-11.

${ }^{10}$ Bialecki, R., Kassab, A., and Fic, A., "Proper Orthogonal Decomposition and Modal Analysis for Acceleration of Transient FEM Thermal Analysis," International Journal for Numerical Methods in Engineering, Vol. 62, No. 6, 14 Feb. 2005 , pp. 774797.

${ }^{11}$ Chandrupatla, T., and Belegundu, A., Introduction to Finite Elements in Engineering, $3^{\text {rd }}$ ed., Prentice-Hall, Upper Saddle River, NJ, 2002, Chap. 5.

${ }^{12}$ Craig, R. Jr., Kurdila, A., Fundamentals of Structural Dynamics, $2^{\text {nd }}$ ed., John Wiley \& Sons, Inc., Hoboken, NJ, 2006, Chaps. 7, 16.

${ }^{13}$ Vosteen, L.F., "Effect of Temperature on Dynamic Modulus of Elasticity of Some Structural Alloys." NACA Technical Note 4348, Langley Aeronautical Laboratory, Langley Field, Virginia, Aug. 1958. 